\title{
Characterization of Gain-of-function Mutant Provides New Insights into ClpP Structure
}

Tengfeng $\mathrm{Ni}^{\S, \dagger, \perp}$, Fei $\mathrm{Ye}^{\ddagger, \neq, \perp}$, Xing $\mathrm{Liu}^{\Sigma, \perp}$, Jie Zhang ${ }^{\S, \perp}$, Hongchuan Liu ${ }^{\S}$, Jiahui $\mathrm{Li}^{\S,+}$, Yingyi Zhang ${ }^{\natural}$, Yingqiang Sun ${ }^{\Delta}$, Meining Wang ${ }^{\Sigma}$, Cheng Luo $^{\neq}$, Hualiang Jiang ${ }^{\neq{ }^{\dagger}}$, Lefu $\operatorname{Lan}^{\S,{ }^{+}}$, Jianhua Gan" ${ }^{\|}$Ao Zhang $^{\Sigma,{ }^{+}}$, Hu Zhou ${ }^{\Sigma,{ }^{+}}$, Cai-Guang Yang ${ }^{\S,+}{ }^{+}$*

${ }^{\S}$ Laboratory of Chemical Biology, State Key Laboratory of Drug Research, Shanghai Institute of Materia Medica, Chinese Academy of Sciences, Shanghai 201203, China;

'University of Chinese Academy of Sciences, Beijing 100049, China;

${ }^{\ddagger}$ College of Life Sciences, Zhejiang Sci-Tech University, Hangzhou 310018, China;

${ }^{\neq}$Drug Design and Discovery Center, State Key Laboratory of Drug Research, Shanghai Institute of Materia Medica, Chinese Academy of Sciences, Shanghai 201203, China;

${ }^{\Sigma}$ CAS Key Laboratory of Receptor Research, Shanghai Institute of Materia Medica, Chinese Academy of Sciences, Shanghai 201203, China;

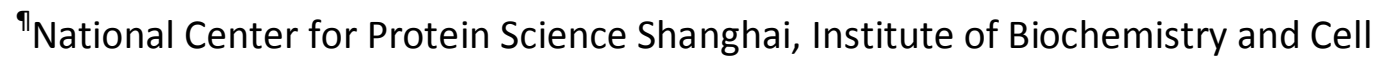
Biology, Shanghai Institute for Biological Sciences, Chinese Academy of Sciences, Shanghai 201210, China;

${ }^{\Delta}$ Experiment center for science and Technology, Shanghai University of Traditional Chinese Medicine, Shanghai 201203, China

"School of Life Sciences, Fudan University, Shanghai 200433, China. 


\section{Supplementary Figures}

A

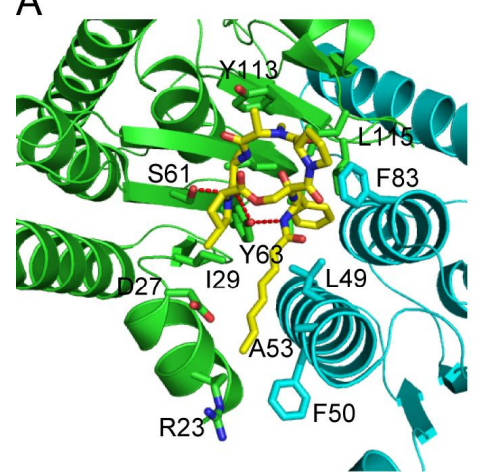

B

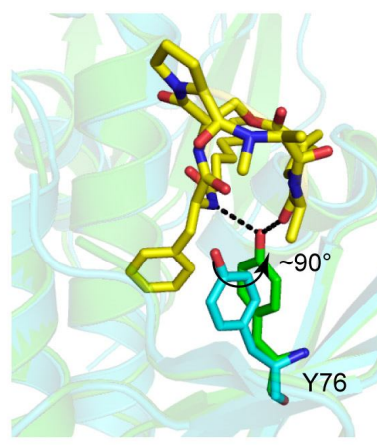

C

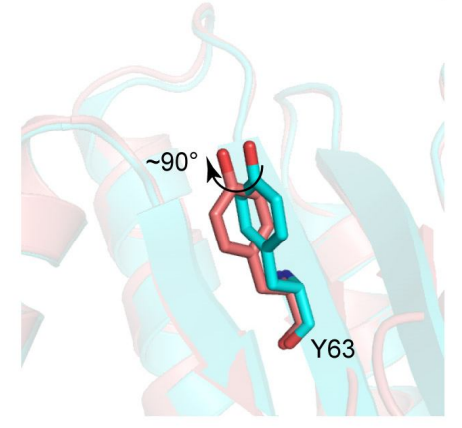

D

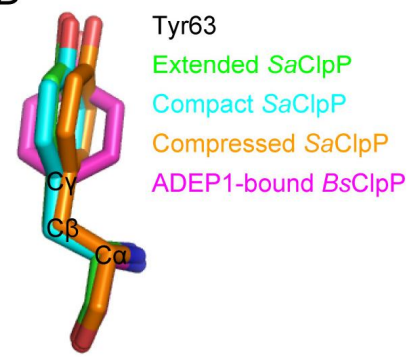

$E$

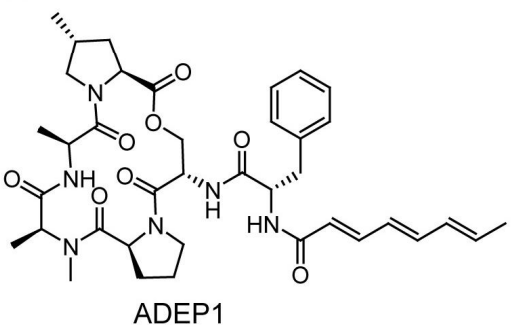

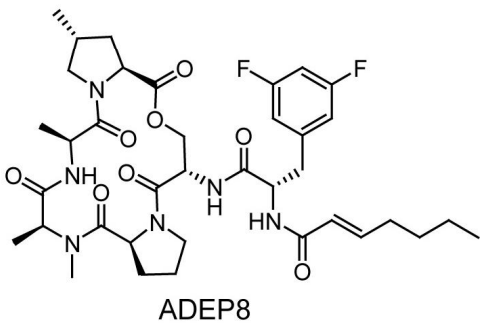

Figure S1. Conformational change of Tyr63 upon ADEPs binding. (A) Detailed interactions between ADEP1 and ClpP from BsClpP. The corresponding tyrosine is Tyr63 in BsClpP. (B) Structural superimposition to show the conformation of Tyr76 of EcClpP in the extended (PDB ID: 1GY6) and ADEP1-bound state (PDB ID: 3MT6), respectively. The ADEP1 is colored in yellow, Tyr76 in ADEP1-bound state in green and apo-extended state in cyan, respectively. The black dashed lines indicate hydrogen bonding. The dihedral angle between the side chains of Tyr76 is about $90^{\circ}$. (C) Structural superimposition of the residue Tyr63 of the MtClpP1 in the extended (PDB code 4UOH) and ADEP4-bound state (PDB code 4UOG), respectively. Tyr63 in ADEP4-bound state is colored in pink and apo-extended state in cyan, respectively. (D) Structural alignments reveal the differential conformations of the side chain of Tyr63 in different states. The extended, compact, and compressed SaClpP is colored in green, cyan, and orange, respectively, and the ADEP1-bound BsClpP is colored in magenta. (E) The chemical structures of ADEP1 and ADEP8 are shown. The known compound ADEP8 is synthesized in our lab, and fully characterized. 
A

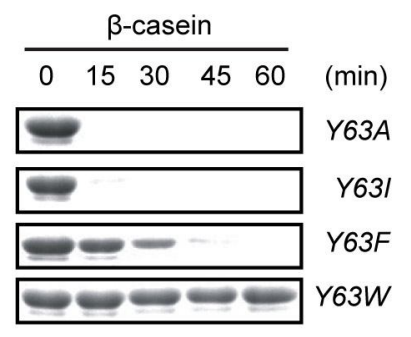

$\mathrm{D}$ $\begin{array}{llllll}M & 0 & 5 & 10 & 30 & 60\end{array}(\mathrm{~min})$

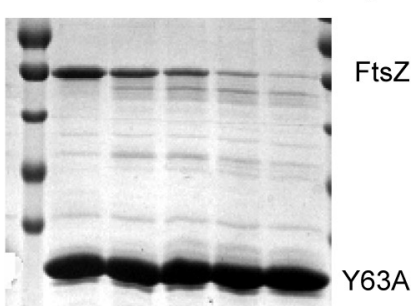

$\mathrm{H}$

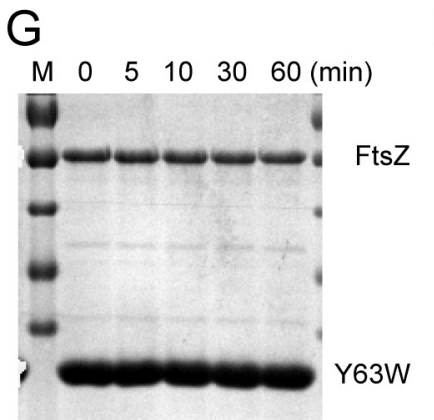

$\mathrm{E}$ $\begin{array}{llllll}M & 0 & 5 & 10 & 30 & 60\end{array}(\min )$

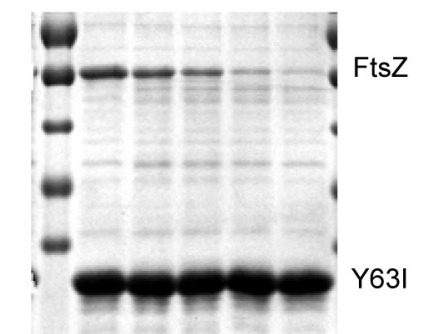

$\mathrm{B}$

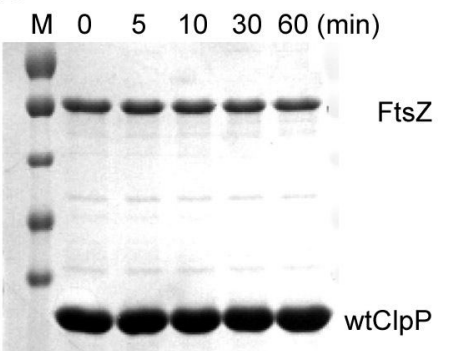

I

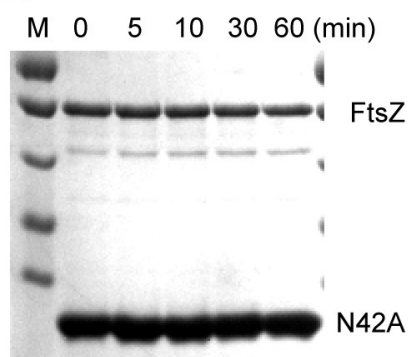

C

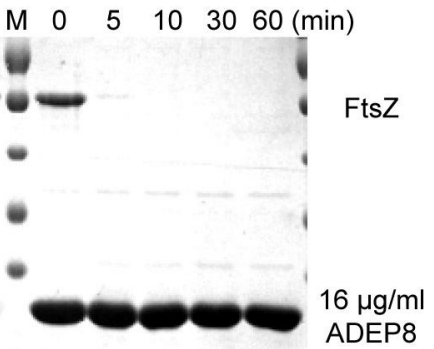

$\mathrm{F}$

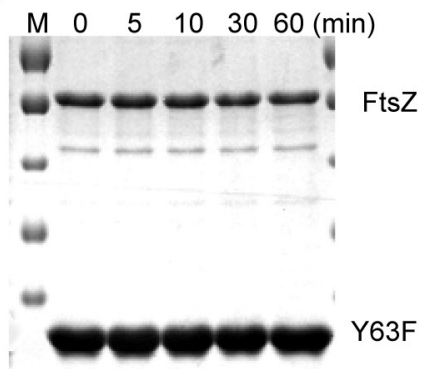
$\begin{array}{llllll}M & 0 & 5 & 10 & 30 & 60\end{array}$ (min)

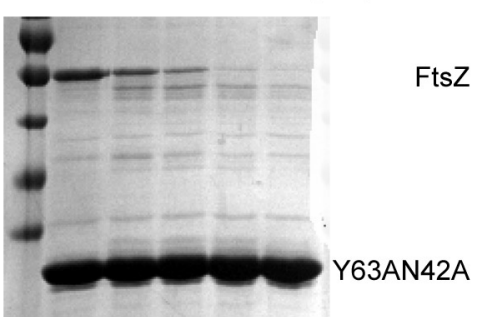

Figure S2. ClpX-independent proteolysis of $\beta$-casein or FtsZ protein by SaClpP variants. The concentration of the SaClpP variants was determined using the bicinchoninic acid (BCA) assay and the same quantity of enzymes were used in these reactions. 
A

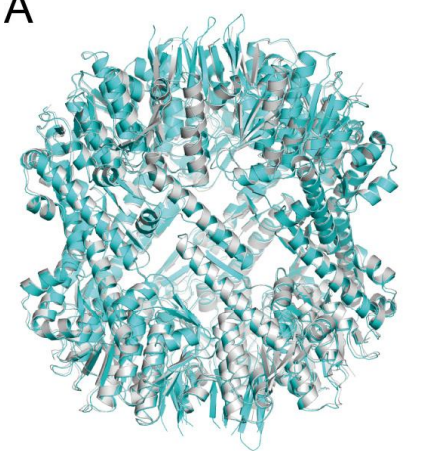

C

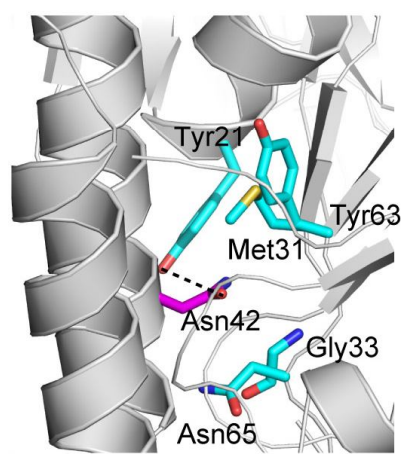

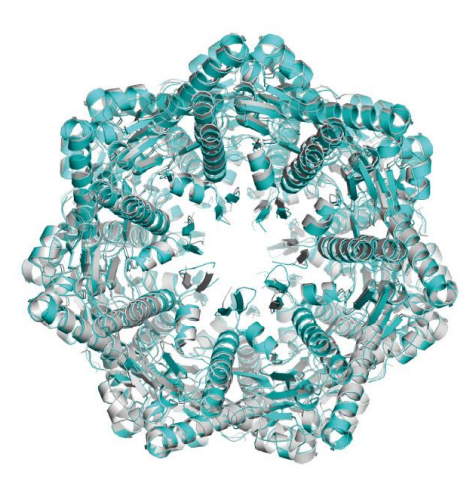

B

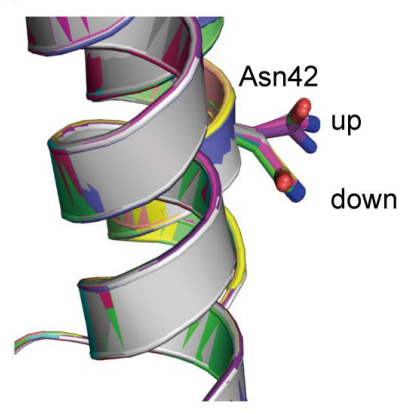

D

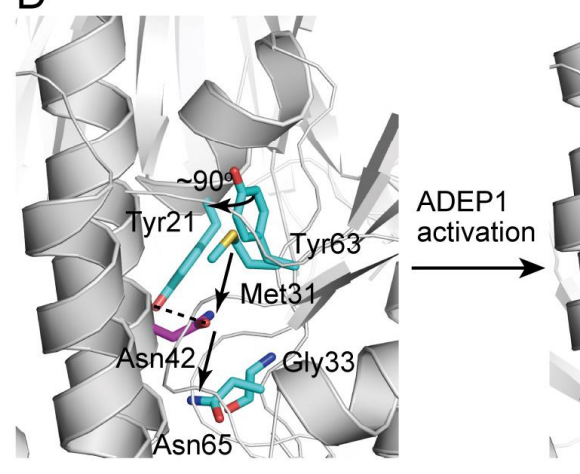

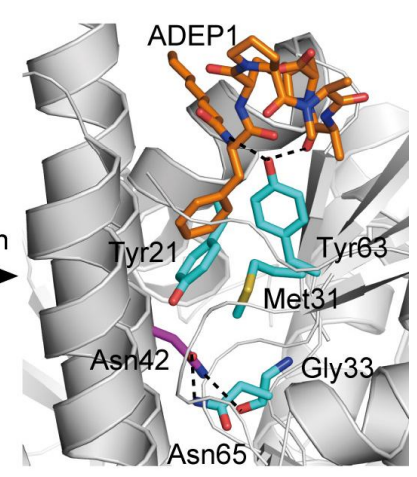

Figure S3. Crystal structure of SaClpPY63A. (A) Structural superimposition of the wild-type SaClpP in the extended state and the SaClpPY63A mutant performed in PyMOL. The extended SaClpP is colored in cyan, and the SaClpPY63A mutant is colored in gray. Two different views of the alignment show that these two structures are highly similar to each other in overall folding. (B) Structural superimposition of all 14 monomers in the SaClpPY63A mutant. Dual conformations of the side chain of Asn42 are observed, either upwards or downwards or both. (C) Close view of the activator-binding site in the extended ClpP. The hydrogen bonding is indicated by black dashed line. (D) Conformational changes in the ADEP1-bound BsClpP. The movements and rotation of side chains are indicated by arrows. The dashed line indicates hydrogen bonding. 
wt

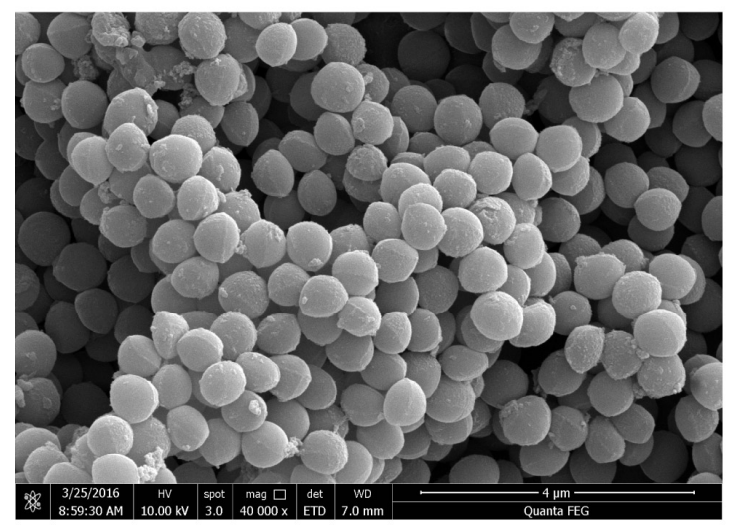

$\Delta c / p P / p Y J 335:: c / p P$

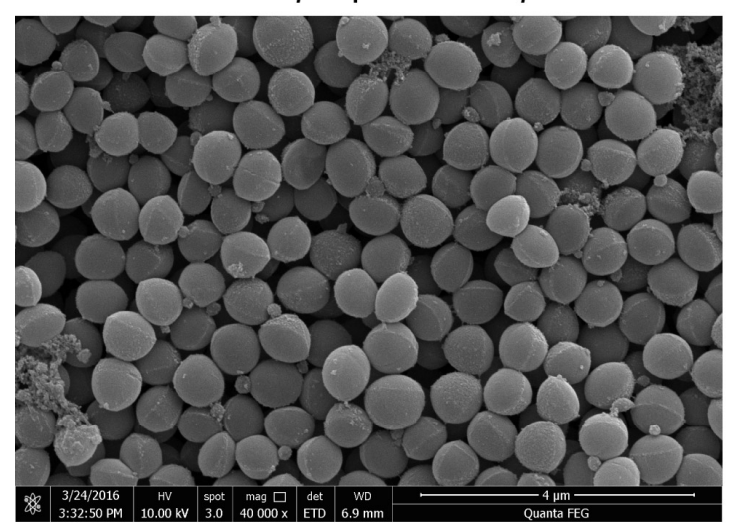

$\triangle c / p P / p Y J 335:: c l p P N 42 A Y 63 A$

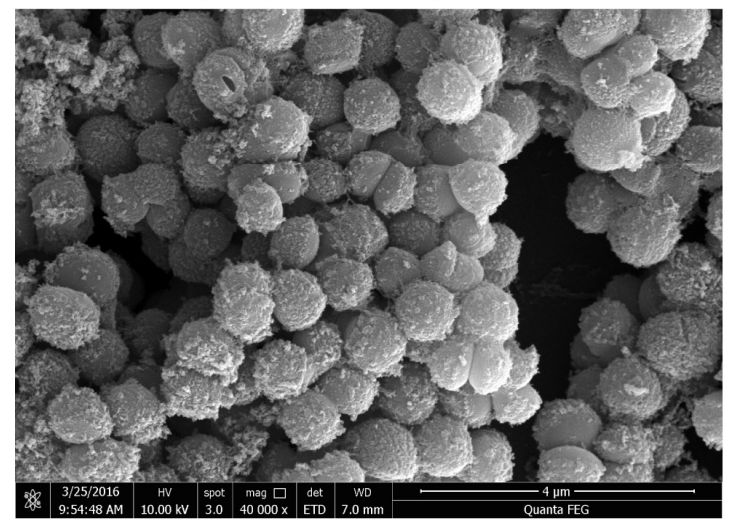

wt/pYJ335

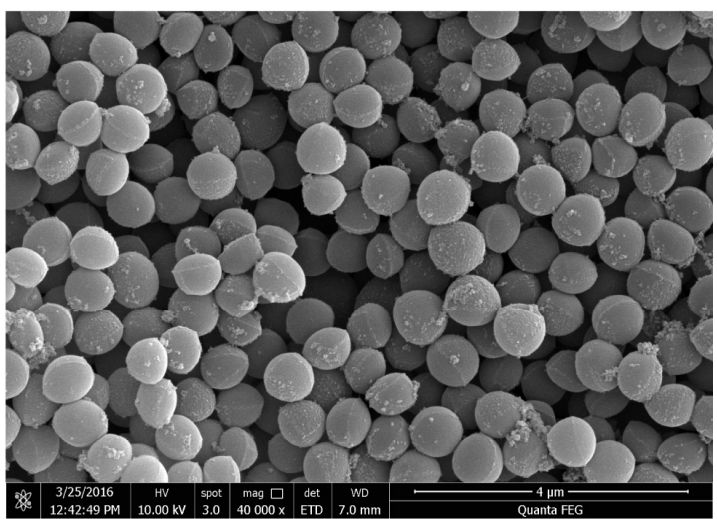

$\Delta c / p P / p Y J 335:: c / p P Y 63 A$

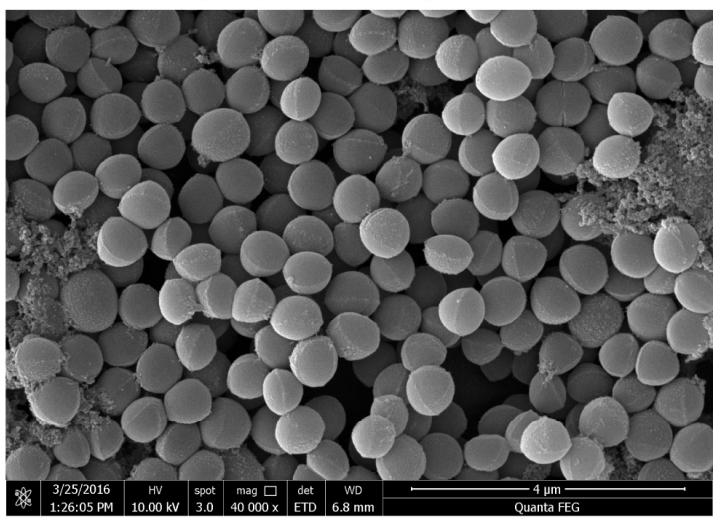

$w t+3 \mu \mathrm{g} / \mathrm{ml}$ ADEP8

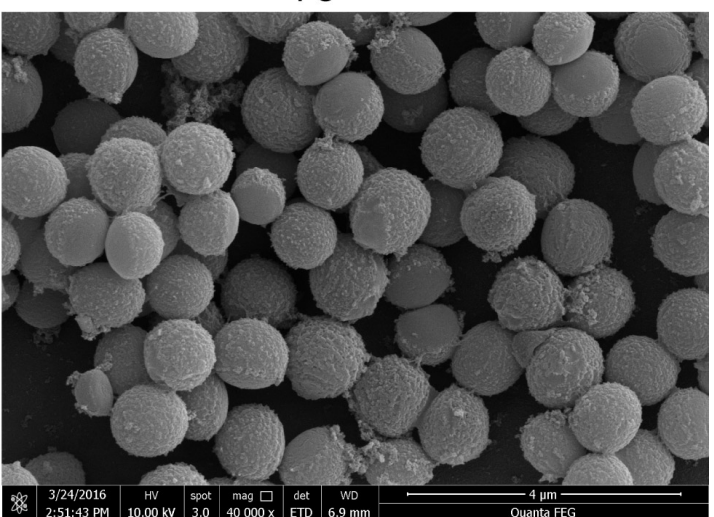

Figure S4. SEM images of $S$. aureus variants are shown. It is presented at magnifications of $\times 40,000$. 


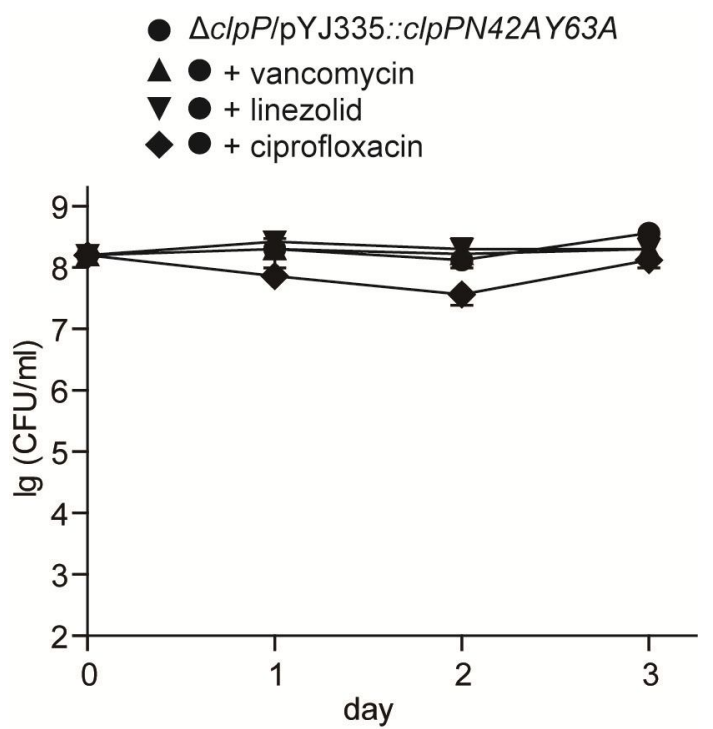

Figure S5. Other antibiotics, such as vancomycin, linezolid, or ciprofloxacin are unable to lead to the eradication of persisters of SaClpPN42AY63A mutant strain after $72 \mathrm{~h}$.

A

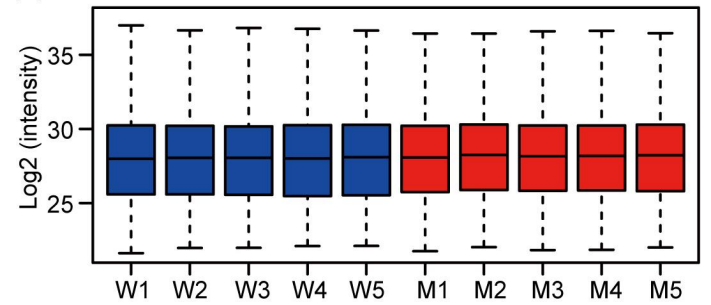

B

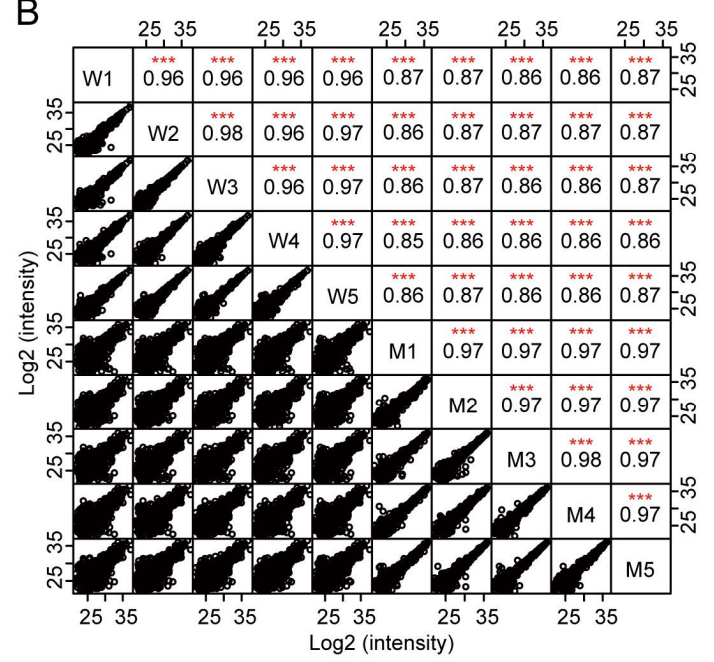

C

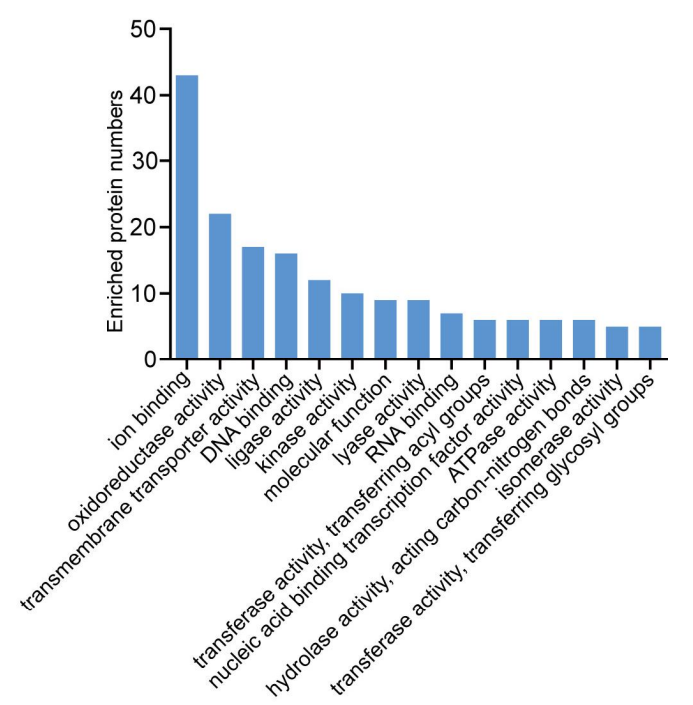

Figure S6. Label-free quantitative proteomic analysis. (A) Box plots of total identified proteins between the wild type control groups (W1-W5) and the allele 
expressing SaClpPN42AY63A groups (M1-M5). (B) A scatter plot and correlation between the control groups and allele groups. (C) Gene ontology analysis of the significantly degraded proteins in the allele expressing SaClpPN42AY63A mutant groups within molecular function annotations.
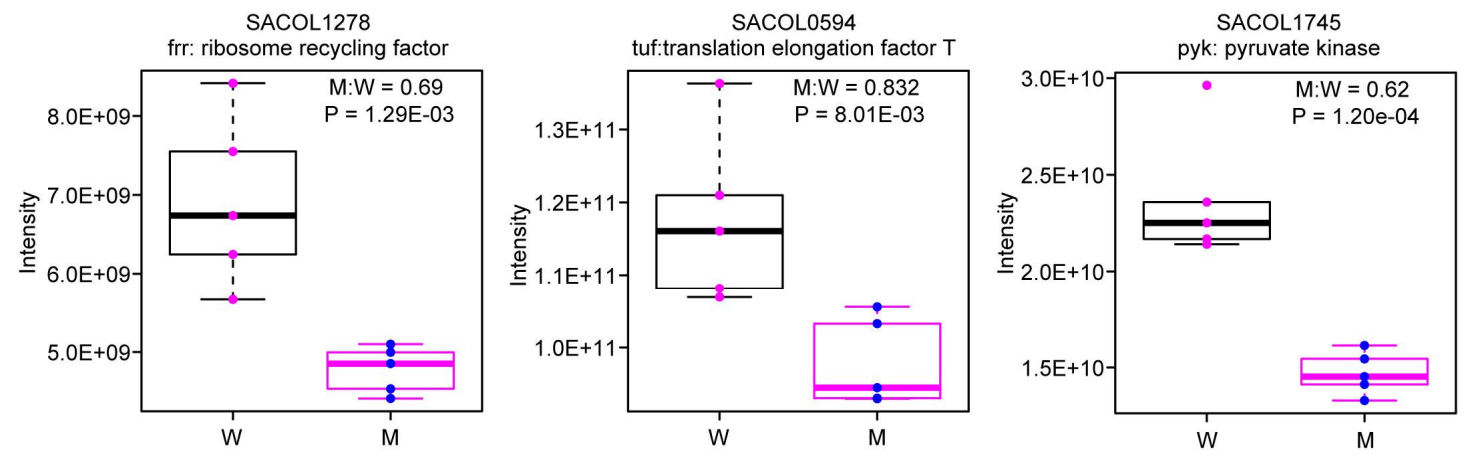

SACOL 0586
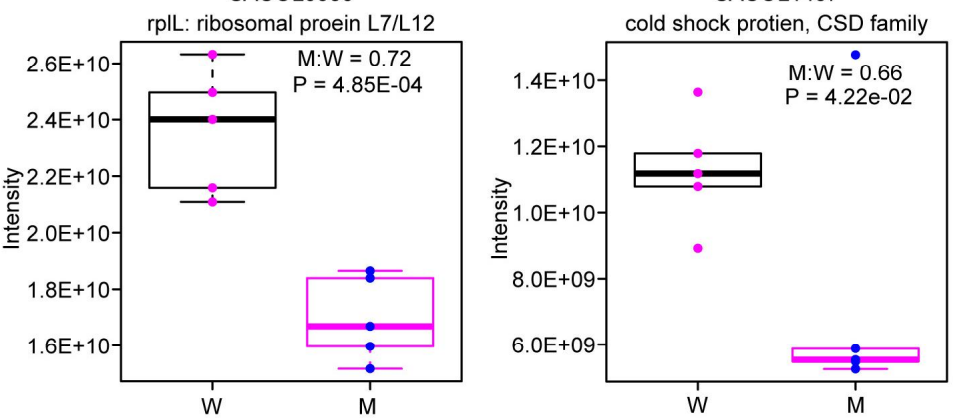

SACOL1632

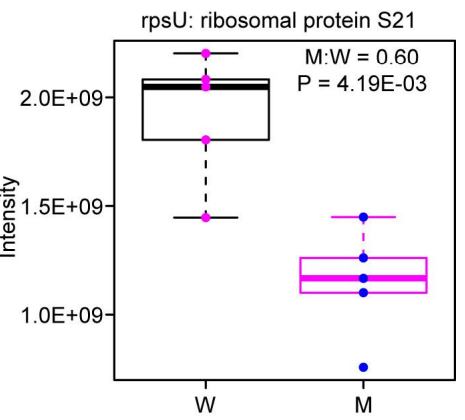

SACOL2214
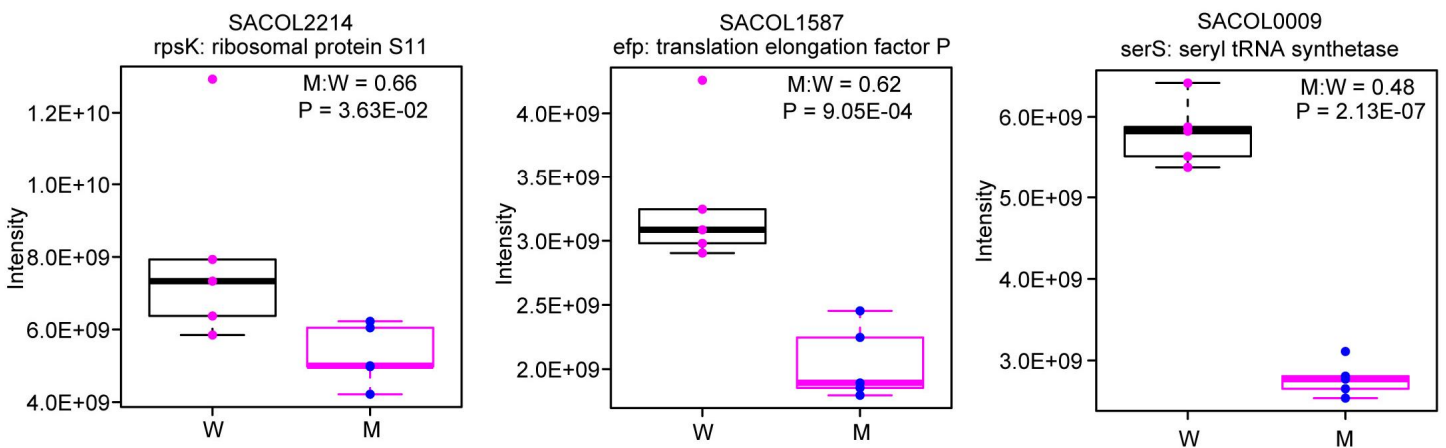

Figure S7. Box plots for the LFQ intensity of overlapped degraded proteins in the allele expressing SaClpPN42AY63A mutant and the previously reported $S$. aureus cells upon ADEP4 treatment. 


\section{Supplementary Table}

Table S1. X-Ray data collection and refinement statistics ${ }^{a}$

\begin{tabular}{|c|c|}
\hline & SaClpPY63A (5C90) \\
\hline \multicolumn{2}{|l|}{ Data collection } \\
\hline Space group & P1 211 \\
\hline \multicolumn{2}{|l|}{ Cell dimensions } \\
\hline$a, b, c(\AA)$ & $94.36,126.24,145.65$ \\
\hline$\alpha, \beta, \gamma\left(^{\circ}\right)$ & $90,93.7,90$ \\
\hline Resolution (Å) & $50.0-1.75(1.81-1.75)$ \\
\hline Total reflections & 1166675 (112177) \\
\hline Unique reflections & 340047 (33993) \\
\hline Rmerge & $0.066(0.325)$ \\
\hline$I / \sigma(I)$ & $16.6(3.8)$ \\
\hline Completeness (\%) & $99.6(100.0)$ \\
\hline Redundancy & $3.4(3.3)$ \\
\hline \multicolumn{2}{|l|}{ Data refinement } \\
\hline Resolution $(\AA ̊)$ & $30.0-1.75(1.795-1.75)$ \\
\hline Reflections (work) & 322791 \\
\hline Completeness (\%) & $99.4(98.3)$ \\
\hline$R_{\text {work }} / R_{\text {free }}$ & $18.2 / 20.2$ \\
\hline \multicolumn{2}{|l|}{ No. atoms } \\
\hline protein & 19924 \\
\hline ligand & 112 \\
\hline water & 1766 \\
\hline \multicolumn{2}{|l|}{ B factors $\left(\AA^{2}\right)$} \\
\hline protein & 27.3 \\
\hline \multicolumn{2}{|l|}{$\mathrm{Rmsd}^{\mathrm{c}}$ in } \\
\hline Bond lengths ( $(\AA)$ & 0.011 \\
\hline Bond angles (ㅇ) & 1.466 \\
\hline \multicolumn{2}{|l|}{ Ramachandran plot ${ }^{d}$} \\
\hline Favoured (\%) & 98.2 \\
\hline Allowed (\%) & 1.7 \\
\hline Unfavoured (\%) & 0.1 \\
\hline
\end{tabular}

${ }^{a}$ The structure was solved using one crystal.

${ }^{\mathrm{b}} \mathrm{Highest}$ resolution shell is shown in parenthesis.

${ }^{c}$ Root mean squared deviation.

${ }^{d}$ Values calculated in CCP4 suite using Procheck. 


\section{Supplementary Methods}

Antibody. The rabbit polyclonal anti-ClpP, anti-FtsZ, and Anti-GAPDH antibody was generated by Shanghai Immune Biotech Ltd using the purified ClpP, FtsZ, and GAPDH protein as the antigen, respectively.

Bacterial Strains and Growth condition. The S. aureus Newman strain was used for antibiotic susceptibility assays, bacterium growth and western blot. Strain was grown at $37^{\circ} \mathrm{C}$ with aeration in tryptic soy broth (TSB, Oxoid). In order to maintain the stability of the plasmid, the medium was supplemented with $10 \mu \mathrm{g} / \mathrm{ml}$ erythromycin if necessary.

Protein Expression and Purification. Wild-type $c l p P$ gene was cloned into vector Pet28b. The expression of the C-His tagged ClpP proteins (SaClpP, BsClpP, and EcClpP) was induced with $0.1 \mathrm{mM}$ IPTG in E. coli BL21 (DE3) Gold strains. The cell pellets were first lysed in buffer A (50 mM Tris- $\mathrm{HCl}, \mathrm{pH} 8.0,100 \mathrm{mM} \mathrm{NaCl}, 50 \mathrm{mM}$ imidazole). The samples were then purified by NI-NTA (GE Healthcare) with the gradient washing by buffer B (50 mM Tris- $\mathrm{HCl}, \mathrm{pH} 8.0,100 \mathrm{mM} \mathrm{NaCl}, 400 \mathrm{mM}$ imidazole). Target eluted fractions were further purified in Superose 200 gel-filtration chromatography (GE Healthcare). ClpP proteins were stocked in buffer C (50 mM Tris- $\mathrm{HCl}, \mathrm{pH} 8.0,100 \mathrm{mM} \mathrm{NaCl}$, and $40 \%$ glycerol) at $-80^{\circ} \mathrm{C}$. All mutations of ClpP were constructed according to the Quikchang Site-Directed mutagenesis Kit (Stratagene), and the similar expression and purification procedures were followed for the production of ClpP mutants. The similar procedures were also applied for the expression and purification of SaFtsz and SaClpX proteins. The protein quantitation was carefully performed by using Pierce BCA Protein Kit (Thermo) in triplicate.

Proteolysis Assays. ATPase-independent proteolysis assay was performed in reaction buffer containing $25 \mathrm{mM}$ HEPES-KOH (pH 7.6), $200 \mathrm{mM} \mathrm{KCl}, 5 \mathrm{mM} \mathrm{MgCl}, 1$ mM EDTA, 10\% (w/v) glycerol, and 2 mM DTT. The reaction was initiated by mixing $15 \mu \mathrm{M} \beta$-casein (Sigma) or $1 \mu \mathrm{M}$ SaFtsZ protein with 1.2 $\mu \mathrm{M}$ SaClpP tetradecamer (or $0.6 \mu \mathrm{M}$ EcClpP tetradecamer, or $1.8 \mu \mathrm{M}$ BsClpP tetradecamer) at $37^{\circ} \mathrm{C}$. Samples 
taken from any single time point were quenched with 1:1 SDS loading buffer immediately and analyzed by $12 \%$ SDS-PAGE with Coomassie Blue Staining. For enzymatic kinetics study, fluorescence-labeled substrate FITC-casein (Sigma) in varying concentrations $(0-40 \mu \mathrm{M})$ was incubated with $0.6 \mu \mathrm{M}$ SaClpP tetradecamer proteins at $37^{\circ} \mathrm{C}$ for $100 \mathrm{~min}$, then fluorescence was recorded every $3 \mathrm{~min}$ on POLARstar Omega (BMG) by using the wavelength of $480 \mathrm{~nm}$ for excitation and 520 $\mathrm{nm}$ for emission, respectively. Reaction velocity of $0-20 \mathrm{~min}$ was used as initial velocity $\left(V_{0}\right)$ for the calculation of $K_{m}$ using GraphPad Prism software (San Diego, USA). For ATPase-dependent digestion assay, $0.6 \mu \mathrm{M}$ SaClpX heptamer was mixed with $5 \mu \mathrm{M}$ GFP-ssrA in reaction buffer containing 25 mM HEPES-KOH (pH 7.6), 200 $\mathrm{mM} \mathrm{KCl}, 5 \mathrm{mM} \mathrm{MgCl}$, $1 \mathrm{mM}$ EDTA, 10\% (w/v) glycerol, and $2 \mathrm{mM}$ DTT. The assay was performed at $37^{\circ} \mathrm{C}$ when $0.3 \mu \mathrm{M} \mathrm{SaClpP}$ or SaClpPY63A was added to the mixture. At each given time point, $50 \mu \mathrm{l}$ sample taken from the assay was mixed with $2 \times$ SDS loading buffer. Results were presented by SDS-PAGE with Coomassie Blue Staining.

Differential Scanning Fluorimetry (DSF). The measurements were performed on the Prism 7500 real-time PCR system (ABI) using SYPRO orange (Invitrogen) as dye. Each sample containing $2 \mu \mathrm{M} \mathrm{ClpP}$ and $5 \times$ SYPRO dye was heated from $25^{\circ} \mathrm{C}$ to $95^{\circ} \mathrm{C}$ at $1 \%$ ramp rate. Fluorescence intensity was monitored at the wavelength of $492 \mathrm{~nm}$ for excitation and $610 \mathrm{~nm}$ for emission, respectively. Melting temperature (Tm) of protein was calculated by Protein Thermal Shift Software (Life Technologies), and further processed in Origin 8.0.

Pull-down Assay. Reactions ( $1 \mathrm{ml}$ ) containing $1.2 \mu \mathrm{M} \mathrm{ClpX}_{6}, 0.6 \mu \mathrm{M} \mathrm{ClpP}_{14}$ and $2 \mathrm{mM}$ ATP $Y$ S were incubated overnight in PD buffer (25 mM HEPES-KOH (pH 7.6), 200 mM $\mathrm{KCl}, 5 \mathrm{mM} \mathrm{MgCl}, 1 \mathrm{mM}$ EDTA, $10 \%(\mathrm{w} / \mathrm{v})$ glycerol, and $2 \mathrm{mM} \mathrm{DTT})$ at $4^{\circ} \mathrm{C}$ with shaking. Samples were gone through $1 \mathrm{ml}$ Ni-NTA (GE Healthcare) and washed with $10 \mathrm{ml}$ PD buffer. Ni-NTA bound proteins were eluted with $10 \mathrm{ml}$ PD buffer containing $200 \mathrm{mM}$ imidazole. The samples were concentrated by filtration to similar volumes, and run on $12 \%$ SDS-PAGE with Coomassie Blue Staining. This experiment was performed in triplicate. 
Plasmid Construction for Constitutive Expression of ClpP Mutants. The $c / p P$ gene from S. aureus Newman strain was constructed into broad-host-range vector pYJ335 which holds a tetracycline-inducible promoter upstream of $c / p P$ gene, thus yielding plasmid pYJ335::clpP. The pYJ335::clppY63A, pYJ335::clpPN42A and pYJ335::clpPN42AY63A mutants were established by PCR with the extension of $7 \mathrm{~min}$ at $68^{\circ} \mathrm{C}$ for 18 cycles according to QuikChange II site-directed mutagenesis kit (Stratagene). All plasmids were transformed into $\triangle c l p P$ Newman strain with the pulse of $2.5 \vee$ for $5.8 \mathrm{~ms}$.

Growth Curve Measurement. S. aureus Newman strains (wt/pYJ335, $\Delta c / p P$, $\Delta c / p P / p Y J 335:: c / p P, \triangle c / p P / p Y J 335:: c / p P Y 63 A)$ were overnight cultured in TSB medium then diluted into an $A_{600}$ of 0.01 . All strains were incubated at $37^{\circ} \mathrm{C}$ with aeration at $230 \mathrm{rpm}$ for $12 \mathrm{~h}$. The absorbance at $600 \mathrm{~nm}$ was measured every hour using GeneQuant spectrophotometry (GE), and each strain was measured three times.

Plate Growth Assay. Overnight culture of S. aureus Newman strain was diluted 1:1000 with fresh TSB medium and incubated at $37^{\circ} \mathrm{C}$ with aeration at $230 \mathrm{rpm}$ for additional $7 \mathrm{~h}$. The cultures were given 1:10 gradient dilution in turn, and then $10 \mu \mathrm{l}$ drops from each gradient was spotted onto the TSB agar plate supplemented with $0.25 \mu \mathrm{g} / \mathrm{ml}$ Tetracycline and $10 \mu \mathrm{g} / \mathrm{ml}$ Erythromycin. Bacteria were counted after incubation at $37^{\circ} \mathrm{C}$ for $10 \mathrm{~h}$.

Western Blot. Bacteria were cultured in TSB medium at $37^{\circ} \mathrm{C}$ with aeration at 225 rpm. $20 \mathrm{ml}$ solution at each given point was taken from each strain and collected by centrifuge at $5000 \mathrm{rpm}$ for $10 \mathrm{~min}$. Sediments were lysised in Tris-EDTA buffer and broken by shaking beads at a speed of $6 \mathrm{~m} / \mathrm{s}$ for $1 \mathrm{~min}$. Sample was made by mixing $200 \mu \mathrm{l}$ total product with $50 \mu \mathrm{l} 5 \times$ SDS loading buffer and heated at $100^{\circ} \mathrm{C}$ for 10 min. The cellular content of target protein was detected by western blot. Samples at each given point were taken from the same growth phase and calibrated by housekeeping protein GAPDH of S. aureus. 
Crystallization, Data Collection, and Structure Determination. Crystallization of purified SaClpPY63A protein was performed using the hanging drop vapor diffusion method at $20^{\circ} \mathrm{C}$. Typically, the crystallization drop was prepared by well mixing a 2 $\mu \mathrm{l}$ protein [4 mg/ml in $20 \mathrm{mM}$ Tris- $\mathrm{HCl}(\mathrm{pH} 8.0), 100 \mathrm{mM} \mathrm{NaCl}$ ] and an equal volume of reservoir solution, and then equilibrated against $500 \mu \mathrm{l}$ of reservoir solution. Hexagonal rod-like crystals appeared in the reservoir solution of $30 \%(w / v)$ 2-methyl-2,4-pentanediol (MPD), $100 \mathrm{mM}$ sodium acetate trihydrate ( $\mathrm{pH} \mathrm{4.8),} \mathrm{and}$ $20 \mathrm{mM}$ calcium chloride dihydrate. The crystals were mounted and flash-frozen in liquid $\mathrm{N}_{2}$ following cryoprotection with the reservoir solution containing an extra 10 20\% MPD. Diffraction data were collected at Shanghai Synchrotron Radiation Facility (SSRF) beamline 17U. All X-ray data were processed using HKL2000 program suite (1) and converted to structure factors within the CCP4 program (2). The crystal belongs to the space group P 121 1. The structures were phased by molecular replacement in Phaser (3), using the extended SaClpP monomer (PDB code 3STA) as the search model. The SaClpPY63A model was built in COOT (4) and the computational refinement was carried out with the program REFMAC5 (5) in the CCP4 suite. Molecular graphics were prepared in PyMOL (6).

Size Exclusion Chromatography. The gel-filtration was conducted at $4{ }^{\circ} \mathrm{C}$ on Superdex 200 HR 10/300 column (GE Healthcare). All ClpP samples were prepared at concentration of $1 \mathrm{mg} / \mathrm{ml}$ in $25 \mathrm{mM}$ HEPES-KOH (pH 7.6), $200 \mathrm{mM} \mathrm{KCl}, 5 \mathrm{mM} \mathrm{MgCl}$, $1 \mathrm{mM}$ EDTA, $10 \%(\mathrm{w} / \mathrm{v})$ glycerol, and $2 \mathrm{mM}$ DTT. $500 \mu \mathrm{l}$ samples were loaded to the column and eluted at a speed of $1 \mathrm{ml} / \mathrm{min}$.

Cryo-Electron Microscopy. All samples were diluted to a concentration of $0.5 \mathrm{mg} / \mathrm{ml}$ for SaClpP, SaClpPN42AY63A, and SaClpP/ADEP8 complex. The SaClpP/ADEP8 complex was assembled by mixing SaClpP monomer $(0.5 \mathrm{mg} / \mathrm{ml}, 50 \mathrm{mM}$ Tris- $\mathrm{HCl}(\mathrm{pH}$ 8.0), $100 \mathrm{mM} \mathrm{KCl}$ ) previously incubated on ice for $30 \mathrm{~min}$ with ADEP8 (20 mM in $100 \%$ DMSO) at 1:5 ratios. For cryo-experiments, $3 \mu$ l of each sample was loaded onto a holey carbon copper grid. The grids were transferred to a Tecnai $\mathrm{G}^{2}$ F20 field emission gun electron microscope operated at $200 \mathrm{kV}$ at liquid nitrogen temperature. 
The images were recorded under a low-does condition ( 20 electrons $\left./ \AA^{2}\right)$ and at a defocus range of $\sim 2-3 \mu \mathrm{m}$ using a FEI Eagle $4 \mathrm{k}$ by $4 \mathrm{k}$ CCD camera. The images of about 2000 particles were reconstructed by EMAN2 program. The inner diameters of 50 particles were manually read in Image $\mathrm{J}$ software, and the average diameter and deviation were determined.

Antibiotic Susceptibility Assays. Stationary phased strains, the wild-type S. aureus Newman, $\Delta c / p P, \Delta c / p P / p Y J 335:: c / p P, \triangle c / p P / p Y J 335:: c l p P Y 63 A$, and $\triangle c / p P / p Y 3335:: c / p P N 42 A Y 63 A$ were cultured in Mueller-Hinton Broth (MHB) at $37^{\circ} \mathrm{C}$ with aeration at $225 \mathrm{rpm}$. Strains containing plasmid PYJ335 were supplemented with $10 \mu \mathrm{g} / \mathrm{ml}$ erythromycin. Antibiotics were added to each target strain in the following concentration: ADEP8 $5 \mathrm{mg} / \mathrm{ml}$, rifampicin $0.4 \mathrm{mg} / \mathrm{ml}$, vancomycin 10 $\mathrm{mg} / \mathrm{ml}$, and linezolid $10 \mathrm{mg} / \mathrm{ml}$ at the stationary-phased growth. The $100 \mu \mathrm{l}$ solution taken from each culture was centrifuged at 12,000 rpm for $1 \mathrm{~min}$, and sediment was resuspended by $100 \mu \mathrm{l}$ PBS. After a series of dilutions, $10 \mu \mathrm{l}$ drops from each gradient was spotted onto the MHB ager plat. Live cell numbers were calculated after 15 -hour incubation at $37^{\circ} \mathrm{C}$.

Scanning Electron Microscopy (SEM). Overnight culture of S. aureus Newman Strains (wt, wt/pYJ335, $\Delta c / p P / p Y J 335:: c l p P, \Delta c / p P / p Y J 335:: c l p P Y 63 A$, $\triangle c / p P / p Y J 335:: c l p P N 42 A Y 63 A$, and wt with $3 \mathrm{ug} / \mathrm{ml}$ ADEP8) grown in $5 \mathrm{ml}$ TSB supplemented with $0.25 \mu \mathrm{g} / \mathrm{ml}$ Tetracycline and $10 \mu \mathrm{g} / \mathrm{ml}$ Erythromycin were diluted into an $A_{600}$ of 0.01 . When all strains were incubated at $37^{\circ} \mathrm{C}$ with aeration at $230 \mathrm{rpm}$ for $7 \mathrm{~h}, 1 \mathrm{ml}$ solution was taken from each strain and collected by centrifuge at $14,000 \mathrm{rpm}$ for $5 \mathrm{~min}$, suspended in $150 \mu \mathrm{l}$ PBS and $10 \mu \mathrm{l}$ was mounted on the surface of sterile glass slides. Adhering bacteria were fixed with $2.5 \%$ glutaraldehyde in PBS for $2 \mathrm{~h}$, washed with $0.1 \mathrm{M}$ sodium cacodylate, post fixed with $1 \%$ osmium tetroxide in PBS for $1 \mathrm{~h}$. Progressive ethanol (30\%, 50\%, 70\%, 90\%, and 100\%) dehydration was performed, followed by specimen critical point drying. Slides were coated with gold-palladium by Leica EM ACE600 and observed under a scanning electron microscope (Quanta FEC 250). The bacteria diameters of 1000 particles 
were manually read in Image $\mathrm{J}$ software, and the average diameter and deviation were determined.

Quantitative Proteomic Analysis. Stationary phase cultures of Newman cells were collected and resuspended in SDT buffer (100 mM Tris-HCl, 100 mM DTT, 4\% SDS, pH 7.6). The cells were lysed in a FastPrep-24 high speed homogenizer (MP Biomedicals) at the following settings: speed, 6.0 ; time, $60 \mathrm{~s}$. This procedure was performed in triplicate. Insoluble material was removed by centrifugation at $16,000 \mathrm{~g}$, and the supernatant was digested using the FASP procedure as previously described $(7,8)$. The digested peptides were submitted to liquid chromatography-tandem mass spectrometry (LC-MS/MS) analysis in annano-ESI quadrupoleExactive (Q Exactive) mass spectrometer (Thermo Fisher Scientific). Peptides were loaded on a $75 \mu \mathrm{m} \times$ $150 \mathrm{~mm}$ fused silica pre-column packed in-house with $3 \mu \mathrm{m}$ ReproSil-Pur C18 beads (120 Å; Dr. MaischGmbH, Ammerbuch, Germany) using an Easy nano-UPLC1000 (Thermo Electron, Waltham, MA). Mobile phase A consisted of $0.1 \%$ formic acid and Mobile phase $B$ consisted of $0.1 \%$ formic acid in acetonitrile. The mass spectrometer was operated in data-dependent mode with each full MS scan, followed by MS/MS for the 15 most intense ions with the parameters: $\geq+2$ precursor ion charge, $2 \mathrm{Da}$ precursor ion isolation window, and $27 \%$ normalized HCD collision energy. The dynamic exclusion was 30s. A full MS scan was collected for peptides from 350 to $1700 \mathrm{~m} / \mathrm{z}$ on the Orbitrap analyzer at a resolution of 70000 (at $\mathrm{m} / \mathrm{z}=200$ ), and the subsequent MS/MS analyses were performed in the same analyzer at a resolution of 17500 (at $\mathrm{m} / \mathrm{z}=200$ ). Maxquant (9) (http://maxquant.org/, version 1.5.1.0) was used to generate peak lists from raw files, and Andromeda (10) was used to search the protein sequence database. Carbamidomethyl (C) was set as a fixed modification, and oxidation $(\mathrm{M},+15.99492 \mathrm{Da})$ was set as a variable modification. Acquired MS/MS spectra were searched against Staphylococcus aureus COLNC 002951 (2673 sequences) protein database from NCBI. The precursor mass tolerances for the first and main searching were set at 20 and $6 \mathrm{ppm}$, respectively, and the fragment mass tolerance for HCD MS/MS spectra was set at $20 \mathrm{ppm}$. Trypsin/P was selected as the 
digestive enzyme with two potential missed cleavages. The false discovery rate (FDR) for peptides and protein groups was rigorously controlled to be $<1 \%$. FDR was calculated by the number of hits from the reverse database divided by the number of forward hits. Label-free quantification was carried out in MaxQuant using intensity determination and normalization algorithm. The "LFQ intensity" of each protein in different samples was calculated as the best estimate, satisfying all of the pairwise peptide comparisons, and this LFQ intensity was almost on the same scale of the summed-up peptide intensities (11). Perseus software (version 1.5.0.15) was used to further process the peptide intensity and protein LFQ intensity lists of the 10 samples. The $p$-value of LFQ intensity of each protein between the two groups by two-tailed Student's t-test using the Perseus program, and $p$ value $<0.05$ and 2 -fold change was set as a cutoff criterion for a significant change.To investigate the significantly changed proteins patterns in specific pathways, the Blast2Go program was used to locate the proteins in the Kyoto Encyclopaedia of Genes and Genomes (KEGG) pathway database (www.genome.jp/kegg). The molecular function annotations of the significantly changed proteins were initially assigned using the Blast2Go program. BLASTp searches were first performed against the NCBI protein database and further analyses included gene ontology (GO) and enzyme code (EC) annotations.

\section{Supplementary Reference}

1. Otwinowski, Z., and Minor, W. (1997) Processing of X-ray diffraction data collected in oscillation mode, pp 307-326, Elsevier.

2. CCP4. (1994) The CCP4 suite: programs for protein crystallography, Acta crystallographica 50, 760-763.

3. Read, R. J. (2001) Pushing the boundaries of molecular replacement with maximum likelihood, Acta crystallographica 57, 1373-1382.

4. Emsley, P., and Cowtan, K. (2004) Coot: model-building tools for molecular graphics, Acta crystallographica 60, 2126-2132.

5. Murshudov, G. N., Vagin, A. A., and Dodson, E. J. (1997) Refinement of macromolecular structures by the maximum-likelihood method, Acta crystallographica 53, 240-255.

6. DeLano, W. L. (Delano Scientific, Palo Alto, CA, 2002). 
7. Wisniewski, J. R., Zougman, A., Nagaraj, N., and Mann, M. (2009) Universal sample preparation method for proteome analysis, Nat Methods 6, 359-362.

8. Chen, Q., Zhang, A., Yu, F., Gao, J., Liu, Y., Yu, C., Zhou, H., and Xu, C. (2015) Label-free proteomics uncovers energy metabolism and focal adhesion regulations responsive for endometrium receptivity, J Proteome Res 14, 1831-1842.

9. Cox, J., and Mann, M. (2008) MaxQuant enables high peptide identification rates, individualized p.p.b.-range mass accuracies and proteome-wide protein quantification, Nat Biotechnol 26, 1367-1372.

10. Cox, J., Neuhauser, N., Michalski, A., Scheltema, R. A., Olsen, J. V., and Mann, M. (2011) Andromeda: a peptide search engine integrated into the MaxQuant environment, J Proteome Res 10, 1794-1805.

11. Cox, J., Hein, M. Y., Luber, C. A., Paron, I., Nagaraj, N., and Mann, M. (2014) Accurate proteome-wide label-free quantification by delayed normalization and maximal peptide ratio extraction, termed MaxLFQ, Mol Cell Proteomics 13, 2513-2526. 